\title{
Integrating genetic algorithms and tabu search for unit commitment problem
}

\author{
M. Sudhakaran' ${ }^{1}$ P. Ajay-D-Vimal Raj ${ }^{2 *}$ \\ ${ }^{I}$ Department of Electrical and Electronics Engineering, Pondicherry Engineering College, Pondicherry University, Pondicherry, India \\ $2^{2 *}$ Department of Electrical and Electronics Engineering, Pondicherry Engineering College, Pondicherry University, Pondicherry, India \\ E-mails:(karan_mahalingam@yahoo.com (M. Sudhakaran); ajayvimal@yahoo.com (P.Ajay-D-Vimal Raj) *Corresponding author)
}

\begin{abstract}
Optimization is the art of obtaining optimum result under given circumstances. In design, construction and maintenance of any engineering system, Engineers have to take many technological and managerial decisions at several stages. The ultimate goal of all such decisions is to either maximize the desired benefit or to minimize the effort or the cost required. This paper shows a memetic algorithm, a real coded Genetic Algorithm combined with local search, synergistically combined with Tabu Search is effective and efficient for solving large Unit Commitment (UC) problems in electrical power systems. A set of feasible generator schedule is first formulated by real coded genetic algorithm method. Then these pre-committed schedules are optimized by ordinary local search and tabu search. The tabu search based hybrid GT algorithm was tested on reported UC problems previously addressed by some existing techniques such as Dynamic Programming (DP), and Simple Genetic Algorithms. Numerical results for systems up to 36 units are given and commented on. A comprehensive unit commitment software package is developed. The future scope of the paper using the proposed method finds a place in the conclusion.
\end{abstract}

Keywords: Unit commitment, economic load dispatch, memetic algorithm, hybrid GT algorithm, local search, tabu search, combinatorial optimization

\section{Introduction}

Optimization, the best way of doing things, is obviously of great interest in the practical world of engineering. In recent years, for power system management, many important decisions are made by describing the system under study as precisely and quantitatively as possible, selecting some measures of system effectiveness, and then seeking the state of the system which gives the most desirable solution to the criteria. Modern electric power systems built with nonlinear characteristics are highly interconnected with wide geographical distribution. This demands the optimization of a complex objective function under few practical constraints. Hence power system network optimization involves maximization or minimization of objective function under certain constraints.

An efficient unit commitment plays an important role in the economic operation of a power system. The objective of unit commitment is to determine when to start up and shut down units such that the total operating cost can be minimized. The standard unit commitment problem is formulated subject to several constraints that include minimum up-time and down-time, crew constraints, ramp rate limits, generation constraints, load balances, must-run units and spinning reserve constraints.

The ideal method of solving the generator scheduling problem involves an exhaustive trial of all the possible solutions and then choosing the best amongst these. This straightforward method would test all combinations of units that can supply the load and reserve requirements. The combination that has the least operating cost is taken as the optimal schedule. Given enough time this enumerative process is guaranteed to find the optimal solution but the solution must be obtained within a time that makes it useful for the intended purpose. Even when the problem is highly constrained, the efficiency of the solution is poor except for the simplest of cases.

Several researches have been done in the unit commitment field for the past two decades. Many papers have recently provided a complete literature synopsis of the solution methods for unit commitment problems. These methods include priority list (Wood and 
Wollenberg, 1984), dynamic programming (Tong et. al., 1991), integer programming (Michaelwich, 1990), branch and bound (Wood and Wollenberg, 1984), linear programming (Tong et. al., 1991), network flow programming (Tong et. al., 1991), lagrangian relaxation (Cheng et. al., 2000) and simulated annealing (Mantawy et. al., 1999). All these methods only provide near global optimal solutions and the quality of each solution is affected by either the solution time limitation, or the feasibility of the final solution. Computer storage requirements used to be another major limiting factor but this is fast becoming a thing of the past as the cost of computer memory continues to drop. Recently there is an upsurge in the use of methods such as Genetic Algorithms and artificial neural networks that mimic natural processes to solve complex problems. Genetic Algorithms have become increasingly popular in recent years in science and engineering disciplines. Some works have been published covering the solution of the unit commitment problem using GA (Maifeld and Sheble, 1996). Solution coding and fitness functions are the most important issues in solving problems using GA. In the literature, unit commitment solutions coding have been done in the binary form. The fitness function has been constructed as the summation of the objective function and penalty terms for constraints violations. The Tabu Search is a powerful optimization procedure that has been successfully applied to a number of combinatorial optimization problems (Mantawy et. al., 1999). It has the ability to avoid entrapment in local optima by employing a flexible memory system. In this paper, a simple TS algorithm based on short-term memory has been proposed for solving the unit commitment problem (Glover and Laguna, 2000). Maifeld and Sheble (1996) have presented a genetic-based unit commitment (UC) scheduling algorithm. It has made use of GA with domain specific mutation operators for finding good unit commitment schedules. The test results of three different electric utilities have been compared with that of Lagrangian relaxation UC method. Bakirtzis et al. (1996) have developed a genetic algorithm that uses different quality function techniques to solve the unit commitment problem. The test results up to 100 generator units have been compared with that of dynamic programming and Lagrangian relaxation methods. Swarup et al. (2002) have employed a new solution methodology to the UC problem using genetic algorithm. The strategy has been found to be efficient and serve to handle larger size UC problems.

Gaing (2003) has built an integrated approach of discrete binary particle swarm optimization (BPSO) with the lambda-iteration method for solving the UC problem. It has been solved as two subproblems using BPSO method for minimization of the transition cost. The economic dispatch problem has been solved by lambda-iteration method for the minimization of the production cost. The feasibility of the method has been demonstrated on a 10- and a 26-unit system, and the test results have been compared with that of GA method. Zhao et al. (2006) have presented an improved particle swarm optimization (IPSO) algorithm for power system UC problem. It has adopted an orthogonal design in order to generate the initial population that are scattered uniformly over a feasible solution space. The IPSO algorithm has been tested on a modeled 10-unit system and the performance is compared with that of GA and EP methods. Ting et al. (2006) have integrated a new approach of hybrid particle swarm optimization (HPSO) scheme, which is a blend of HPSO, BPSO and real-coded particle swarm optimization (RCPSO), to solve the UC problem. The UC problem has been handled by BPSO, whereas the economic load dispatch problem has been solved by RCPSO. Funabashi et al. (2007) have formulated a twofold simulated annealing method for the optimization of fuzzy-based UC model. The method has served to offer a robust solution for UC problem. Victoire et al. (2005) have applied a hybrid PSO and sequential quadratic programming (SQP) technique, prelude to tabu search (TS) method for solving the UC problem. The combinational part of the UC problem has been solved using the TS method. The nonlinear optimization part of economic dispatch problem (EDP) has been solved using a hybrid PSO-SQP technique. The effectiveness of hybrid optimization technique has been tested on a NTPS zone-II 7-unit system. Grey and Sekar (2008) have presented a unified solution of the SCUC using linear programming (LP) as the optimization tool and an extended DC network model for accounting for security and contingency concerns and for calculating the ED. An extended DC model that includes line flows permits a more practical approach to include security consideration.

Unlike the traditional SCUC algorithms presented in the literature, the proposed unified method solves the optimization from the network outward to the generation schedule. In this paper, we propose a new hybrid GT algorithm for solving the UC problem. The algorithm integrates the main features of the genetic algorithm based Memetic Algorithm and Tabu Search. The paper is organized as follows: In section 2, the problem formulation for unit commitment with all constraints is presented. The proposed Hybrid GT Algorithm, which is a genetic algorithm, combined with local search and Tabu Search is given in section 3. Implementations of GA and TS in proposed Hybrid GT algorithm are shown in section 4 and section 5 respectively. Test cases and simulation results are discussed in section 6 and conclusions and the future scope of the paper are summarized in section 7 .

\section{Problem formulation}

The generator scheduling problem involves the determination of the start up / shut down times and the power output levels of all the generating units at each time step, over a specified scheduling period $T$, so that the total start up, shut down and running costs are minimized subject to system and unit constraints.

The fuel cost, $F C_{i}$ per unit in any given time interval is a function of the generator power output. A frequently used cost function is

$$
F C_{i}=A_{i} P_{i}^{2}+B_{i} P_{i}+C_{i} \quad \mathrm{Rs} / \mathrm{Hr}
$$

$A_{i}, B_{i}, C_{i}$ represent unit cost coefficients, while $P_{i}$ is the unit power output. 
The Generator start up cost depends on the time the unit has been off prior to start up. The start up cost in any given time interval can be represented by an exponential cost curve

$$
S C_{i}=\sigma_{i}+\delta_{i}\left\{1-\exp \left(-T_{o f f, i} / \tau_{i}\right)\right\}
$$

$\sigma_{i}$ is the hot start up cost, $\delta_{i}$ the cold start up cost, $\tau_{i}$ the unit cooling time constant and is $T_{o f f i}$ the time a unit has been off. The shut down cost, $\mathrm{SD}$ is usually given a constant value for each unit. The total production cost, $F_{T}$ for the scheduling period is the sum of the running cost, start up cost and shut down cost for all the units.

$$
F_{T}=\sum_{i=1}^{T} \sum_{i=1}^{N} F C_{i, t}+S C_{i, t}+S D_{i, t}
$$

The overall objective is to minimize $F_{T}$ subject to a number of constraints]:

(i) System hourly power balance, where the total power generated must supply the load demand $P_{D}$ and system losses $P_{L}$,

$$
\sum_{i=1}^{N} P_{i} u_{i}-\left(P_{D}+P_{L}\right)=0 \quad t=1,2 \ldots \ldots \ldots . . T
$$

(ii) Hourly spinning reserve requirements $\mathrm{R}$ must be met

$$
\sum_{i=1}^{N} P_{i}^{\max } u_{i}-\left(P_{D}+P_{L}\right)=R \quad t=1,2 \ldots \ldots \ldots T
$$

(iii) Unit rated minimum and maximum capacities must not be violated

$$
P_{i}^{\min } \leq P_{i} \leq P_{i}^{\max }
$$

(iv) The initial unit states at the start of the scheduling period must be taken in to account.

(v) Minimum up / down (MUT / MDT) time limits of units must not be violated,

$$
\begin{aligned}
& \left(T^{o n}{ }_{t-1, i}-M U T_{i}\right)\left(u_{t-1, i}-u_{t, i}\right) \geq 0 \\
& \left(T^{o f f}{ }_{t-1, i}-M D T_{i}\right)\left(u_{t, i}-u_{t-1, i}\right) \geq 0
\end{aligned}
$$

$T_{\text {off }} / T_{\text {on }}$ is the unit off / on time, while $u_{t, i}$ denotes the unit off / on [0,1] status. Other constraints such as unit rate limits, crew constraint limitations, unit status restrictions, unit derating factors can be considered but are not included in this paper.

\section{GA implementation in the proposed GT algorithm}

The details of the implementation of GA components are summarized here as follows:

\subsection{Coding of solution}

The solution in the unit commitment problem is represented by a binary matrix $(U)$ of dimension $T \mathrm{X} N$. The proposed method for coding is a mixer of binary and decimal numbers. Each column vector in the solution matrix (Which is the operation schedule of one unit) of length $T$ is converted to its equivalent decimal number. The solution matrix is then converted into one row vector (chromosome) of $N$ decimal numbers $(U 1, U 2, \ldots U N)$, each represents the schedule of one unit. The numbers $U 1, U 2, \ldots U N$ are integers ranging from 0 to $2^{N}-1$. Accordingly, a population of size $N P O P$ is stored in a matrix $N P O P \mathrm{X} N$.

\subsection{Fitness function}

As we are generating always feasible solutions in the proposed GT algorithm, the fitness function is taken as the reciprocal of the total production cost. The fitness function is then scaled to prevent the premature convergence. Linear scaling is used in our algorithm, which requires linear relationship between the original fitness function and the scaled one (Michaelwich, 1990). 


\subsection{Crossover}

In this paper the window crossover operation is performed between two chromosomes in their binary form. Applying window crossover operator to parents $X$ and $Y$, and obtaining offspring $Z$, works as follows:

(i) Set parent $X$ to have fitness $(X)>$ fitness $(Y)$

(ii) Sample 1 from uniform $(0, \mathrm{~T}), \mathrm{w}$ from uniform $(0, \mathrm{~N}), \mathrm{u}$ from uniform $(1, N-w)$, and $\mathrm{h}$ from uniform $(1, T-1)$ distributions.

(iii) Define a window $W_{1}$, of dimension $1 w$, with left upper corner $[u, 1]$ and right lower corner $[u+w, h+1]$.

(iv) Define a window $W_{2}$ as the complement of $W_{1}$.

(v) If (dimension of $W_{1}$ is greater than dimension of $W_{2}$ ) Copy genes of $X\left[W_{1}\right]$ to $Z\left[W_{1}\right]$ and genes from $Y\left[W_{2}\right]$ to $Z\left[W_{2}\right]$.

Else

Copy genes of $X\left[W_{2}\right]$ to $Z\left[W_{2}\right]$ and genes from $Y\left[W_{1}\right]$ to $Z\left[W_{1}\right]$.

\subsection{Mutation}

Mutation operation is performed by randomly selecting chromosome with a prespecified probability. The selected chromosome is decoded to its binary equivalent. Then the unit number and time period are randomly selected and the rule of mutation is applied to reverse the status of units keeping the feasibility of the constraints Sudhakaran et al. (2002).

\subsection{Swap Mutation Operator}

After performing crossover and mutation, the swap mutation operator is added. The swap operator uses the full load average costs $(A F L C)$ of the generating units to perform a swap of unit states. The $A F L C$ of a unit is defined as the cost per unit of power when the generator is at its full capacity. When the fuel cost is given by the equation $F C_{i}=A_{i} P_{i}^{2}+B_{i} P_{i}+C_{i} \mathrm{Rs} / \mathrm{Hr}$

$A F L C$ can be expressed as

$$
A F L C_{\mathrm{i}}=C_{\mathrm{i}} / P_{i}^{\max }+B_{i}+A_{i} P_{i}^{\max }
$$

The generating units are ranked by their $A F L C$ in ascending order. Units with lower $A F L C$ should have higher priority to be dispatched. At a given hour, the operator probabilistically swaps the states of two units $i$ and $j$ only if the unit $i$ is ranked better than unit $j(i<j)$ and the state of the units are 'off' and 'on', respectively. This operator is a modified version of the priority list dispatching heuristic, where the generating units are committed in a predetermined order.

\section{TS Implementation in the proposed GT algorithm}

Tabu Search is characterized by an ability to escape local optima by using a short-term memory of recent solutions. This is achieved by a strategy of forbidding certain moves. The purpose of classifying certain moves as forbidden - i.e. "tabu" is basically to prevent cycling. Moreover, TS permits backtracking to previous solutions, which may ultimately lead, via a different direction, to better solutions.

The main two components of TS algorithm are the Tabu List (TL) restrictions and Aspiration Level (AV) of the solution associated with the recorded moves. They are discussed in the following sections.

\subsection{Tabu List (TL)}

TL is managed by recording moves (trial solutions) in the order in which they are made. Each time a new element is added to the "bottom" of a list, the oldest element on the list is dropped from the "top". TL sizes, which provide good results, often grow with the size of the problem and stronger restrictions are generally coupled with smaller sizes. Best sizes of TL lie in an intermediate range between these extremes. In some applications a simple choice of TL size in a range centered around 7 seems to be quite effective.

\subsection{Aspiration Criteria (AV)}

Another key issue of TS arises when the move under consideration has been found to be tabu. Associated with each entry in the tabu list there is a certain value for the evaluation function called Aspiration Level (AV). Roughly speaking, AV criteria are designed to override tabu status if a move is "good enough".

In the proposed algorithm TS is used to generate new neighbors to randomly selected members of the GA populations. The flow chart of Figure 2 describes the main step of the TS algorithm. 


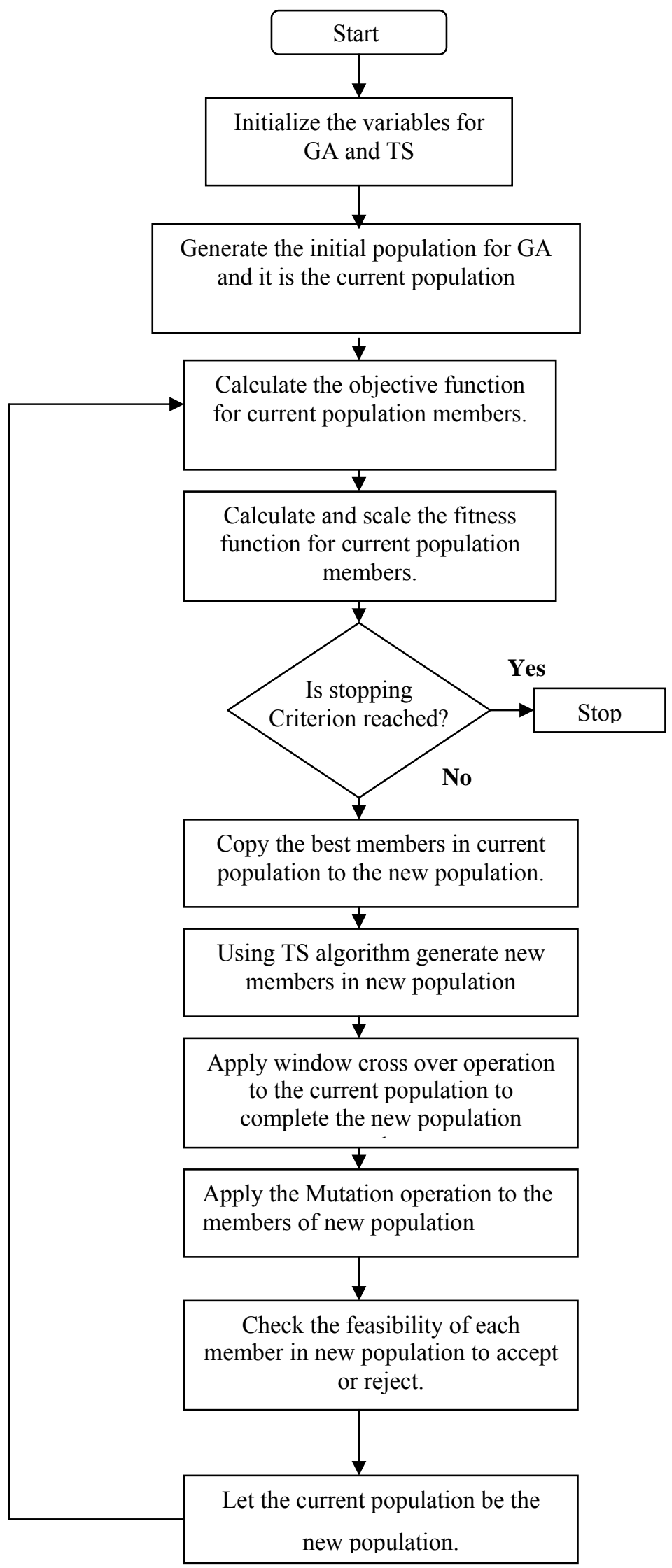

Figure 1. Flowchart of proposed hybrid GT method 


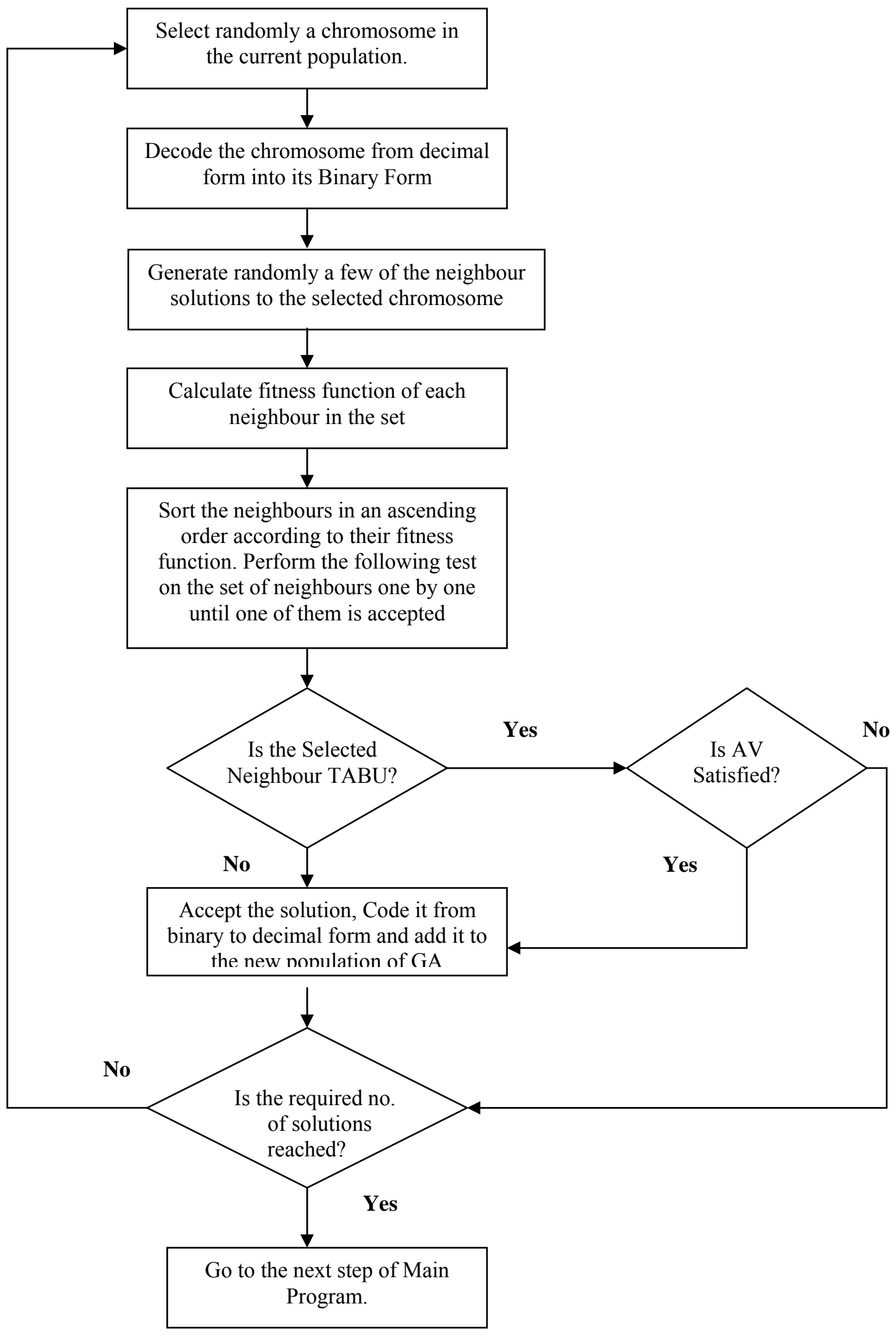

Figure 2. Flow chart of Tabu search part 


\section{Local Search}

At each generation, two local searches 1-opt and 2-opt, were applied to the best solution of the new generation. 1-opt flips one bit of the solution matrix, whereas 2-opt switches the status of two bits. The 2-opt operator first searches for improvement by switching the status of two units at each hour. Then, the search continues by switching the status of a unit at two different hours. As soon as a better solution is found the local search is stopped and the modified solution replaces the original solution in the new generation.

\section{Proposed hybrid GT algorithm}

In solving the Unit Commitment problem, status of a unit $i$ at hour $t\left(u_{t, i}\right)$ and start-up/shut-down status of unit $i$ at hour $t\left(v_{t, i}\right)$ which are binary variables, and the units output power variables $\left(P_{t, i}\right)$ which are continuous variables need to be determined. The first is a combinatorial optimization problem while the second is a nonlinear one. A hybrid of GA and TS is proposed to solve the combinatorial optimization. The Economic Load Dispatch (ELD) is solved by lambda iterative technique. The proposed hybrid GT algorithm differs from other evolutionary computing techniques in providing an acceptable solution within a relatively short time. In this technique in phase 1, the genetic algorithm samples a large search space, climbs many peaks in parallel, and is likely to lead the search towards the most promising solution area. In phase 2, Tabu search is used to narrow down the global optimal solution area, and the local searches 1-opt and 2-opt are used to tune the method towards the global optimal solution. Figure 1 gives the flow chart of the proposed hybrid GT algorithm

The algorithm has the procedural steps as follows:

1. Initial population of $\mathrm{np}$ trial solutions is generated. Each solution is taken as a real vector with their dimensions corresponding to the number of control variables.

2. From the expression (5) the fitness function value is evaluated for each solution vector.

3. The termination condition is checked. If the termination condition is reached, the process is stopped, if not the next step is pursued.

4. The best members of the current population are copied to the new population.

5. Tabu search algorithm is used to generate new members in the new population (up to $15 \%$ ) as neighbours to randomly selected solutions in the current population.

6. The window crossover operator is applied to complete the members of the new population

7. Non-uniform mutation operator is applied to the new population

8. 1-opt and 2-opt local searches are applied for the fine tuning of the solution space to get the global optimal solution

9. The members of the new population are tested so as to treat them as members of the current population. Then the process is continued from step- 2 .

The tabu search part of the proposed algorithm is shown in Figure 2 and the steps are explained as follows,

1. A chromosome is randomly selected from the current population and decoded from decimal to binary form.

2. For the selected chromosome a few neighbour solutions are randomly generated and their fitness function values are calculated.

3. The neighbours are sorted in an ascending order according to their fitness value and the following test is performed on the set of neighbours one by one until one of them is accepted.

4. The selected neighbour is checked. If it is a TABU, the satisfaction of aspiration value (AV) is checked. If it is not a TABU, the solution is accepted and step 6 is pursued.

5. If AV is satisfied, the solution is accepted and step 6 is pursued. Otherwise the neighbour is rejected and the next step is taken.

6. If the required number of solutions is reached, the process returns to the main program. Otherwise it will go to step 1.

Stopping Criteria

There are several possible stopping conditions for the search. In our hybrid algorithm, we stop the process if one of the following two conditions is satisfied:

- The number of iterations performed since the best solution last changed is greater than a predefined maximum number of iterations, (or)

- If maximum allowable number of iterations (generations) is reached.

\section{Test problems}

In order to test the validity of the proposed hybrid GT algorithm, the results of five constrained optimization problems have been considered Baskar and Subburaj (2000) All the problems are test cases to solve nonlinear optimization problems. For the proposed method the parameters were selected as: Population size $=20$, Crossover probability $=0.6$, Mutation probability $=0.02$, the 
maximum number of generations $=500$, TL size $Z=7$. All the programs were developed using MATLAB package. Every test case was solved for 50 individual trials on Pentium IV computer with $2.5 \mathrm{GHz}$ for the proposed Hybrid GT algorithm. For the purpose of comparison of performance of different algorithms, the results of Hybrid algorithm (Kim and Myung, 1997), CADE algorithm (Storn, 1999) and Real coded GA (Baskar and Subburaj, 2000) are taken directly from the sources.

\author{
Test problems: \\ Problem 1: $\quad$ Minimize $\mathrm{f}(x)=100\left(x_{2}-x_{1}^{2}\right)^{2}+\left(1-x_{1}\right)^{2}$ \\ Subjected to the nonlinear constraints \\ $\mathrm{C} 1: x_{1}+x_{2}^{2} \geq 0$ \\ $\mathrm{C} 2: x_{1}^{2}+x_{2} \geq 0$ \\ And bounds $-0.5 \leq x_{1} \leq 0.5$ and $x_{2} \leq 1.0$ \\ Problem 2 : $\quad$ Minimize $\mathrm{f}(x)=-x_{1}-x_{2}$ \\ The known global solution is $\mathrm{f}(0.5,0.25)=0.25$.
}

Subject to the nonlinear constraints

C1: $x_{2}-2 x_{1}^{4}+8 x_{1}^{3}-8 x_{1}^{2} \leq 2$

C2: $x_{2}-4 x_{1}^{4}+32 x_{1}^{3}-88 x_{1}^{2}+96 x_{1} \leq 36$ and

Bounds $0 \leq x_{1} \leq 3$ and $0 \leq x_{2} \leq 4$

The known global solution is $\mathrm{f}(2.32952,3.17849)=-5.508014$.

Table 6. gives the comparison of results for the 5 test cases. From the above case study it is true that GA is spending most of its time competing between different hills, rather than improving the solution along a single hill on which the optimal solution is located. Table 7 shows the comparison of execution time taken by all the algorithms for solving the test cases. From the comparison tables and figures it is proved that the proposed hybrid GT algorithm is performing better than other algorithms both in terms of reliability and computation efficiency. In this paper, a new integrated Hybrid Genetic Tabu search algorithm is proposed to solve the combinatorial optimization problems. When the problem is highly nonlinear, this algorithm out performs other algorithms in terms of the quality of the solution and computation expenses. In phase 1 of the proposed algorithm, the real coded GA is used to narrow down the solution space and in phase 2 the Tabu search is used along with the local searches to locate the global optimal solution. This hybrid algorithm handles either inequality or equality constraints and the feasible region do not have to be convex and gradient or other auxiliary informations are not required. In order to demonstrate the effectiveness of the proposed algorithm, this method is applied to five constrained optimization test problems and the results obtained are compared with other methods. From the study it is clearly proved that the proposed Hybrid GT algorithm is superior and it can achieve over other conventional algorithms and hybrid algorithms.

\title{
8. Power system examples
}

In order to test the proposed hybrid algorithm, the following four examples from the literature were considered.

(i) 4 unit system (Saneifard et.al., 1997)

(ii) 8 unit system (Sudhakaran et al., 2000)

(iii) 10 unit system (Cheng et. al., 2000)

(iv) 36 unit system (Ma and Shahidehpour, 1999)

The following control parameters have been chosen after running a number of simulations: Population size $=20$, Crossover probability $=0.55$, Mutation probability $=0.01$, the swap mutation probability $=0.3$. The maximum number of generations $=1500$, TL size $Z=7$. For a sample, the data for the 10 unit system is shown in Table 1 and the 24 hr load cycle in Table 2 . The comparisons of results for the above four test cases are shown in Table 3 and Table 5. The schedule obtained by the proposed method for the example problem 3 is shown in Table 4 . The superiority of the proposed algorithm is obvious. It is clear that the Hybrid GT algorithm performs better than the individual algorithms, in terms of both total production cost and execution time.

\section{Conclusion}

The paper solves the unit commitment problem by the Hybrid GT algorithm, which incorporates the concept of Tabu search into the Genetic Algorithm method and it is demonstrated with the numerical examples. For the example problems 1 and 2 , the results obtained from the proposed algorithm were compared with the DP method and GA method. For the example problems 3 and 4 , the results were compared with the Lagrangian relaxation (LR) method and GA method. The convergence of GT algorithm is shown in Figure 3 and the reliability of the proposed method is shown in Figure 4. From the above comparison it is proved that the proposed hybrid GT algorithm can be applied to the unit commitment of large power system with any number of units and it can 
give global optimal solution with lesser computation time. The proposed method should be used for solving the unit commitment problem by taking into account the ramp rate limits as it is a limitation for the amount of power generated per hour, security limits and voltage constraints limit at various buses has to be checked in order to provide a secured power system. Therefore Hybrid GT algorithm can also be tested for a unit commitment problem having ramp rate limits, voltage constraints and security limits. In this approach a hybrid GT based algorithm solves the unit commitment problem. The proposed algorithm integrates the main features of the most commonly used evolutionary methods such as GA and Tabu Search for solving combinational optimization problems. The algorithm is based mainly on the TS whereas the GA method is used to generate new members in the population to guide the search towards the optimal solution. The use of genetic scheme improves the performance of coding the combination of units and to arrange the ON / OFF status of the units. GT method is used for power output estimation and to locate the global optimal solution by fine tuning the search process. The implementation of the proposed method is demonstrated using a test systems and power system examples. The results proved the effectiveness of the algorithm in solving the UC problem with a reduced production cost. In the proposed approach, an integrated genetic algorithm method with tabu search technique has been used to solve the unit commitment problem. The feasibility of the proposed method was demonstrated on a 4,8,10 and 36 unit system and the test results were compared in terms of production cost with those obtained by the GA and DP methods

\section{Acknowledgement}

The authors are very thankful to the authorities of Pondicherry Engineering College, Pondicherry-605 014 for providing all facilities to complete this piece of work.

\section{References}

Baskar S, Subburaj P, 2000. Performance of hybrid real coded genetic algorithms, JIE, Vol. 81, pp 25-32.

Su C.-C.and Hsu Y.-Y., 1991. Fuzzy dynamic programming: an application to unit commitment, IEEE Transactions on Power Delivery, Vol. 6, No.3, pp. $64-65$.

Cheng C.-P., Lie C.-W. and Liu C.-C., 2000. Unit commitment by Lagrangian relaxation and genetic algorithms, IEEE Transactions on Power systems, Vol.15, No.2, pp. 707-714.

Gaing Z.-L., 2003. Discrete particle swarm optimization algorithm for unit commitment, in Proc. 2003 IEEE Power Engineering Society General Meeting, Vol. 1, pp. 418-424.

Glover F. and Laguna M., 1997. Tabu search, Kluwer Academic Publishers, Massachusetts, USA.

Grey and Sekar, 2008, Unified solution of security-constrained unit commitment problem using a linear programming methodology, IET Gener. Transm. Distrib., Vol. 2, No. 6, pp. 856-867.

Juste K.A, Kita H, Tanaka E, and Hasegawa J, 1999. An evolutionary programming solution to the unit commitment problem, IEEE Transactions on Power systems, Vol.14, No.4, pp. 1452-1459.

Kazarlis S. A., Bakirtzis, A. G., and Petridis. V., 1996. A Genetic algorithm solution to the unit commitment problem, IEEE Trans. Power Systems, Vol. 11, No. 1, pp. 83-92.

Kim J.-H.and Myung H., 1997, Evolutionary programming techniques for constrained optimization problems, IEEE Transaction on Evolutionary Computation, Vol.1, No.2, pp 129-140.

Ma H. and Shahidehpour S.M., 1999. Unit commitment with transmission security and voltage constraint, IEEE Transactions on Power Systems, Vol.14, No.2, pp. 757-764.

Maifeld T.T.and Sheble G.B., 1996. Genetic-based unit commitment algorithm, IEEE Transactions on Power systems, Vol.11, No.3, pp. 1359-1370.

Mantawy A.H, Youssef L. Abdel-Magid, Shokri Z. Selim, 1998. Unit commitment by tabu search, IEE proceedings-Generation, Transmission and Distribution, Vol.145, No.1, pp. 56-64.

Mantawy A.H, Youssef L. Abdel-Magid, Shokri Z. Selim, 1999. Integrating GA, TS and SA for the unit commitment problem, IEEE Transactions on Power systems, Vol.14, No.3, pp. 829-836.

Michalewicz Z., 1990, Genetic Algorithm + Data Structure = Evolution Program, Springer Verberg Heidelberg, New York.

Saber A.Y., Senjyu T., Yona A., Urasaki N., and Funabashi T., 2007. Fuzzy unit commitment solution-A novel twofold simulated annealing approach, Electric Power Systems Research, Vol. 77, pp. 1699-1712.

Saneifard S., Prasad N.R., Smolleck H.A.,1997. A fuzzy logic approach to unit commitment, IEEE Transactions on Power Systems, Vol.12, No.2, pp. 988-995.

Storn R., 1999, System design by constraint adaptation and differential evolution, IEEE Transactions on Evolutionary Computation, Vol.3, No.1, pp. 22-34.

Sudhakaran M., Kannan P.S. and Venkatesh P., 2000, Application of computational intelligence to economic load dispatch, JIE, Vol.81, pp 39-43.

Sudhakaran M., Slochanal M.R. and Praba K.B, 2000. Application of fuzzy logic to unit commitment problem, NCIEES-2000, PSG Tech, Coimbatore. 
Sudhakaran M, Slochanal M.R., 2002. Application of refined genetic algorithm to unit commitment problem, National seminar on Effective Utilization of Generated Power, IE chapter, Bhubaneswar, Orissa.

Swarp K. S. and Yamashiro S., 2002. Unit commitment solution methodology using genetic algorithm, IEEE Trans. Power Systems, Vol. 17, pp.87-91.

Ting T. O., Rao .M. V. C., and Loo .2006.C. L., A novel approach for unit commitment problem via an effective hybrid particle swarm optimization, IEEE Trans. Power Systems, Vol. 21, No.1, pp. 411-418.

Tong S.K, Shahidehpour S.M and Ouyang Z, A Heuristic, 1991. Short term unit commitment, IEEE Transactions on Power Systems, Vol. 6, No. 3, pp.1210 - 1216.

Victoire T. A. A. and Jeyakumar A. E., 2005.Unit commitment by a tabu-search-based hybrid-optimization technique, IEE Proc. Generation, Transmission and Distribution, Vol. 152, No .4, pp. 563-574.

Wood A.J. and Wollenberg B.F., 1984. Power Generation, Operation and Control, John Wiley and Sons.

Zhao B., Guo C. X., Bai,. B. R. and Cao Y. J., 2006. An improved particle swarm optimization algorithm for unit commitment, International Journal of Electrical Power and Energy Systems, Vol. 28, No. 1, pp. 482-490.

\section{ANNEXURE}

Table-1 Cost coefficients - 10-unit system

\begin{tabular}{|c|c|c|c|c|c|c|c|c|c|c|}
\hline Particulars & 1 & 2 & 3 & 4 & 5 & 6 & 7 & 8 & 9 & 10 \\
\hline $\operatorname{Pmax}(\mathrm{MW})$ & 455 & 455 & 130 & 130 & 162 & 80 & 85 & 55 & 55 & 55 \\
\hline $\operatorname{Pmin}(\mathrm{MW})$ & 150 & 150 & 20 & 20 & 25 & 20 & 25 & 10 & 10 & 10 \\
\hline $\mathrm{A}(\$ / \mathrm{h})$ & 1000 & 970 & 700 & 680 & 450 & 370 & 480 & 660 & 665 & 670 \\
\hline $\mathrm{B}(\$ / \mathrm{MW}-\mathrm{h})$ & 16.19 & 17.26 & 16.6 & 16.50 & 19.7 & 22.26 & 27.74 & 25.92 & 27.27 & 27.79 \\
\hline $\mathrm{C}\left(\$ / \mathrm{MW}^{2}-\mathrm{h}\right)$ & 0.0004 & 0.0003 & 0.002 & 0.0021 & 0.0039 & 0.0071 & 0.0079 & 0.0041 & 0.0022 & 0.0017 \\
\hline Min up (h) & 8 & 8 & 5 & 5 & 6 & 3 & 3 & 1 & 1 & 1 \\
\hline Min dn (h) & 8 & 8 & 5 & 5 & 6 & 3 & 3 & 1 & 1 & 1 \\
\hline Hot start $\operatorname{cost}(\$)$ & 4500 & 5000 & 550 & 560 & 900 & 170 & 260 & 30 & 30 & 30 \\
\hline Cold start cost $(\$)$ & 9000 & 10000 & 1100 & 1120 & 1800 & 340 & 520 & 60 & 60 & 60 \\
\hline Cold start hrs(h) & 5 & 5 & 4 & 4 & 4 & 2 & 2 & 0 & 0 & 0 \\
\hline Initial status (h) & 8 & 8 & -5 & -5 & -6 & -3 & -3 & -1 & -1 & -1 \\
\hline
\end{tabular}

Table-2 Daily generation - 10-unit system

\begin{tabular}{|c|c|c|c|}
\hline Hour & $\mathrm{P}_{\text {Load (MW) }}$ & Hour & $\mathrm{P}_{\text {Load (MW) }}$ \\
\hline 1 & 700 & 13 & 1400 \\
\hline 2 & 750 & 14 & 1300 \\
\hline 3 & 850 & 15 & 1200 \\
\hline 4 & 950 & 16 & 1050 \\
\hline 5 & 1000 & 17 & 1000 \\
\hline 6 & 1100 & 18 & 1100 \\
\hline 7 & 1150 & 19 & 1200 \\
\hline 8 & 1200 & 20 & 1400 \\
\hline 9 & 1300 & 21 & 1300 \\
\hline 10 & 1400 & 22 & 1100 \\
\hline 11 & 1450 & 23 & 900 \\
\hline 12 & 1500 & 24 & 800 \\
\hline
\end{tabular}


Table 3. Comparison of solution quality

\begin{tabular}{|c|c|c|c|c|}
\hline \multirow{2}{*}{ Units } & \multicolumn{3}{|c|}{ Total production cost (\$) } & \multirow{2}{*}{$\begin{array}{c}\text { Execution } \\
\text { time (Sec) }\end{array}$} \\
\cline { 2 - 5 } & DP method & GA method & $\begin{array}{c}\text { Proposed } \\
\text { GT method }\end{array}$ & 15 \\
\hline 4 & 78358 & 77377 & 77320 & 95 \\
\hline
\end{tabular}

Table 4. UC schedule obtained after final population- 10 Unit system unit

Houx

\begin{tabular}{|c|c|c|c|c|c|c|c|c|c|c|}
\hline & 1 & 2 & 3 & 4 & 5 & 6 & 7 & 8 & 9 & 10 \\
\hline 1 & 1 & 1 & 0 & 0 & 0 & 0 & 0 & $\mathbf{0}$ & 0 & 0 \\
\hline 2 & 1 & 1 & 0 & 0 & $\mathbf{0}$ & 0 & 0 & $\mathbf{0}$ & 0 & 0 \\
\hline 3 & 1 & 1 & 1 & 0 & 0 & 0 & 0 & 0 & 0 & 0 \\
\hline 4 & 1 & 1 & 1 & 1 & 0 & 0 & 0 & $\mathbf{0}$ & 0 & 0 \\
\hline 5 & 1 & 1 & 1 & 1 & $\mathbf{0}$ & 0 & 0 & 0 & 0 & 0 \\
\hline 6 & 1 & 1 & 1 & 1 & 1 & 0 & 0 & 0 & 0 & 0 \\
\hline 7 & 1 & 1 & 1 & 1 & 1 & 0 & 0 & 0 & 0 & 0 \\
\hline 8 & 1 & 1 & 1 & 1 & 1 & 0 & 0 & 0 & 0 & 0 \\
\hline 9 & 1 & 1 & 1 & 1 & 1 & 1 & 1 & 0 & 0 & 0 \\
\hline 10 & 1 & 1 & 1 & 1 & 1 & 1 & 1 & 1 & 0 & 0 \\
\hline 11 & 1 & 1 & 1 & 1 & 1 & 1 & 1 & 1 & 1 & 0 \\
\hline 12 & 1 & 1 & 1 & 1 & 1 & 1 & 1 & 1 & 1 & 1 \\
\hline 13 & 1 & 1 & 1 & 1 & 1 & 1 & 1 & 1 & 0 & 0 \\
\hline 14 & 1 & 1 & 1 & 1 & 1 & 1 & 1 & 0 & 0 & 0 \\
\hline 15 & 1 & 1 & 1 & 1 & 1 & 0 & 0 & 0 & 0 & 0 \\
\hline 16 & 1 & 1 & 1 & 1 & 1 & 0 & 0 & 0 & 0 & 0 \\
\hline 17 & 1 & 1 & 1 & 1 & 1 & 0 & 0 & 0 & 0 & 0 \\
\hline 18 & 1 & 1 & 1 & 1 & 1 & 0 & 0 & 0 & 0 & 0 \\
\hline 19 & 1 & 1 & 1 & 1 & 1 & 0 & 0 & 0 & 0 & 0 \\
\hline 20 & 1 & 1 & 1 & 1 & 1 & 1 & 1 & 1 & 0 & 0 \\
\hline 21 & 1 & 1 & 1 & 1 & 1 & 1 & 1 & 0 & 0 & 0 \\
\hline 22 & 1 & 1 & 1 & 1 & $\mathbf{0}$ & 1 & 1 & 0 & 0 & 0 \\
\hline 23 & 1 & 1 & 1 & 0 & 0 & 0 & 0 & 0 & 0 & 0 \\
\hline 24 & 1 & 1 & 0 & 0 & 0 & 0 & 0 & 0 & 0 & 0 \\
\hline
\end{tabular}


Vol. 2, No. 1, 2010, pp. 57-69

Table 5. Comparison of solution quality

\begin{tabular}{|c|c|c|c|c|}
\hline \multirow{2}{*}{ Units } & \multicolumn{3}{|c|}{ Total production cost (\$) } & \multirow{2}{*}{$\begin{array}{c}\text { Execution } \\
\text { time(Sec) }\end{array}$} \\
\cline { 2 - 4 } & DP Method & GA Method & $\begin{array}{c}\text { Proposed } \\
\text { GT Method }\end{array}$ & 182 \\
\hline 10 & 565825 & 565825 & 561238 & 380 \\
\hline 36 & 1348176 & 1347787 & 1342386 & \\
\hline
\end{tabular}

Table 6. Comparison of results for the test problems

\begin{tabular}{|c|c|c|c|c|c|}
\hline $\begin{array}{c}\text { Problem } \\
\text { No. }\end{array}$ & Exact Optimal & $\begin{array}{l}\text { Best Hybrid } \\
\text { Algorithm } \\
\text { Kim and } \\
\text { Myung (1997) }\end{array}$ & $\begin{array}{c}\text { CADE } \\
\text { Storn (1999) }\end{array}$ & $\begin{array}{c}\text { Real Coded } \\
\text { GA, Baskar } \\
\text { and Subburaj } \\
(2000)\end{array}$ & $\begin{array}{c}\text { Proposed } \\
\text { Hybrid GT } \\
\text { Algorithm }\end{array}$ \\
\hline $\begin{array}{c}\mathbf{b} \\
\mathbf{m} \\
\mathbf{w}\end{array}$ & 0.250000 & $\begin{array}{l}0.250000 \\
0.250000 \\
0.250000\end{array}$ & $\begin{array}{l}0.250000 \\
0.250000 \\
0.250000\end{array}$ & $\begin{array}{l}0.250000 \\
0.250000 \\
0.250000\end{array}$ & $\begin{array}{l}0.250000 \\
0.250000 \\
0.250000\end{array}$ \\
\hline $\begin{array}{c}\mathbf{b} \\
\mathbf{m} \\
\mathbf{w}\end{array}$ & -5.508014 & $\begin{array}{l}-5.508013 \\
-5.508013 \\
-5.508013\end{array}$ & $\begin{array}{l}-5.508014 \\
-5.508014 \\
-5.508014\end{array}$ & $\begin{array}{l}-5.50801 \\
-5.50795 \\
-4.41250\end{array}$ & $\begin{array}{l}-5.508014 \\
-5.508014 \\
-5.508014\end{array}$ \\
\hline
\end{tabular}

b- best; m-mean; w-worst; Results of 50 Independent runs

Table 7 Performance of different algorithms for the test problems

\begin{tabular}{|c|c|c|c|c|}
\hline $\begin{array}{c}\text { Problem } \\
\text { No. }\end{array}$ & $\begin{array}{c}\text { Best Hybrid Algorithm } \\
(\mathrm{Sec})\end{array}$ & $\begin{array}{c}\text { CADE } \\
(\mathrm{Sec}) \\
\text { Storn }(1999)\end{array}$ & $\begin{array}{c}\text { Real Coded GA } \\
(\mathrm{Sec}) \\
\text { Baskar and Subburaj } \\
(2000)\end{array}$ & $\begin{array}{c}\text { Proposed Hybrid GT } \\
\text { Algorithm (Sec) }\end{array}$ \\
\hline 1 & 1.759 & 1.263 & 4.4 & 0.55 \\
\hline 2 & 1.382 & 3.1434 & 4.61 & 0.72 \\
\hline
\end{tabular}

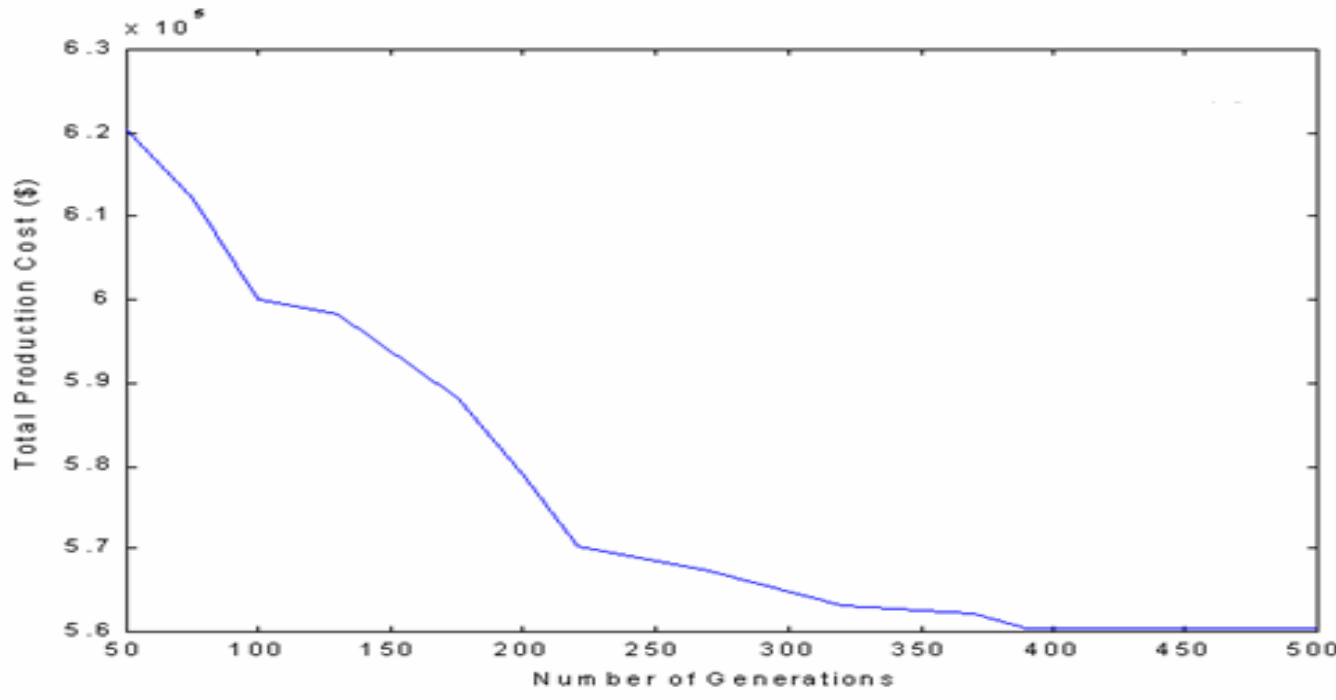

Figure 3 GT convergence characteristics - 10-unit system 


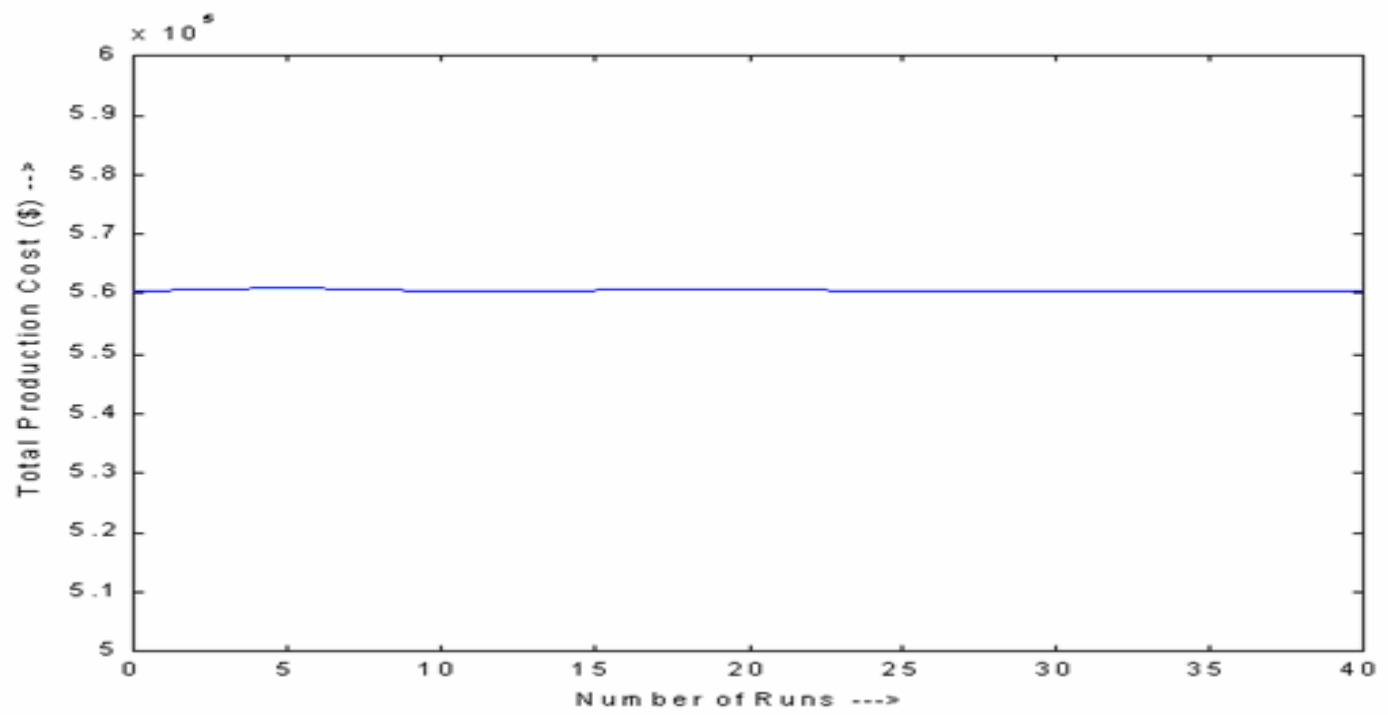

Figure 4 Reliability of GT Method - 10-unit system

\section{Biographical notes}

Dr. M. Sudhkaran is Associate Professor, Department of Electrical and Electronics Engineering, Pondicherry Engineering College, Pondicherry, (India). He has engaged in teaching and research activities since the last 15 years. His field of specialization is Power Systems. Dr. M.S.K. has published several papers in various national, international conferences and journals. He is guiding 6 students for Ph.D. work.

Dr. P. Ajay-D-Vimal Raj is Assistant Professor, Department of Electrical and Electronics Engineering, Pondicherry Engineering College, Pondicherry, (India). He has engaged in teaching and research activities since the last 15 years. His field of specialization is optimization techniques in Power Systems. Dr. Raj has published several papers in various national, international conferences and journals.

Received December 2009

Accepted January 2010

Final acceptance in revised form January 2010 